\title{
Distribution of prime numbers Fundamental Theorem
}

\author{
Dan Liu \\ Department of Mathematics, Chinese sichuan Neijiang Teachers University, Chinese, P.R.China.
}

Keywords: prime number; density; theorem; calculation.

Abstract: In 1849, the German mathematician Gauss large average distribution density of primes near x. Based on the density of the Gauss proposed regional distribution of prime numbers theorem. And regional distribution of prime numbers theorem proved easy to understand way. The fundamental theorem to obtain the distribution of prime numbers. Thus proving that a new prime number theorem. Therefore gives the details of the calculation.

\section{Introduction}

In 1849, the German mathematician Gauss to Encke's letter said that, from 1792 to 1793 by examining the number of prime numbers in the period of 1000 adjacent integer, found a large $\mathrm{x}$ primes average distribution density should as follows:

$$
\frac{1}{\ln x}
$$

by (1), Points [1],[2],[3],[5],[6]:

$$
\pi(x) \sim \operatorname{Li}(x)=\int_{2}^{x} \frac{1}{\ln u} d u, \quad(x \rightarrow \infty) .
$$

This (2) is called: the prime number theorem.

According to Gauss investigation, this paper proposed the primes distribution theorem and proof area. The fundamental theorem to obtain the distribution of prime numbers. This has proved a another prime number theorem.

\section{Gauss density}

Set a large number $\mathrm{x}$, the density of primes the number of $\pi(\mathrm{x}, \mathrm{y})$, get:

$$
\frac{\pi(x, y)}{x-y} \sim \frac{1}{\ln x}, \quad x>y
$$

This (3) as: Gauss density.

For example: Let $\mathrm{x}=100000000, \mathrm{y}=99995200$, by (3) calculated as follows:

Actual density $\frac{\pi(x, y)}{x-y}=\frac{259}{4800} \approx 0.05395833333$,

Gauss density $\frac{1}{\ln x}=\frac{1}{\ln 100000000} \approx 0.0542868102$,

The calculations show that the theory and practice is very close.

$$
\begin{aligned}
& \text { Set } y=x / \lambda, \lambda>1, \text { by (3) get: } \\
& \qquad \pi(x, x / \lambda) \sim \frac{x(\lambda-1)}{\lambda \ln x},
\end{aligned}
$$

Set $x=100000000, \quad \lambda=1.000048$, by (4) Calculate:

The actual number $\pi(\mathrm{x}, \mathrm{x} / \lambda)=259$,

Theoretical number $\frac{x(\lambda-1)}{\lambda \ln x}=260.58$,

The calculation shows that very close to the actual number. However, after all, are not equal. Can be equal? 


\section{The regional distribution of prime numbers theorem}

Set a large number $\mathrm{x}$, the prime number $\mathrm{p}$, the number of prime numbers is $\pi(\mathrm{x}, \mathrm{x} / \lambda)$, get $[7]_{115},[8]_{112}$ :

$$
\pi(x, x / \lambda)=\frac{x(\lambda-1)}{\ln \lambda} \sum_{x / \lambda \leq p \leq x} \frac{1}{\lambda p}, \quad \lambda>1,
$$

This (5) called: Regional distribution of prime numbers theorem. This is the the Gauss density of origin.

For example: Let $\mathrm{x}=100000000, \quad \lambda=1.000048$, by (5) Calculate:

$\pi(x, x / \lambda)=259+0.00028$

Actual $\pi(x, x / \lambda)=259$

The calculations show that the remainder is ignored 0.00028 integer is equal to the actual number 259,

Proof:

Set a large number $\mathrm{x}, \lambda$ Approaching 1, the prime numbe $\mathrm{p}$, Series and an integer, Clear:

$$
\sum_{x / \lambda \leq p \leq x} \frac{x-x / \lambda}{\pi(x, x / \lambda) p \ln \lambda}=1
$$

For example: Let $\mathrm{x}=100000000, \lambda=1.000048$, by (6) and of the series 1.0000010810530266 , To ignore 0.0000010810530266 , integer 1 ,

By (6) get:

$$
\pi(x, x / \lambda)=\sum_{x / \lambda \leq p \leq x} \frac{x-x / \lambda}{p \ln \lambda}
$$

By (7) get:

$$
\pi(x, x / \lambda)=\frac{x(\lambda-1)}{\ln \lambda} \sum_{x / \lambda \leq p \leq x} \frac{1}{\lambda p}
$$

Theorem (5) is proved.

\section{The distribution of prime numbers Fundamental Theorem}

By (5) get:

$$
\begin{aligned}
& \pi(x)=s\left(x, x^{1 / 2}\right)+\pi\left(x^{1 / 2}\right), \\
& s\left(x, x^{1 / 2}\right)=\frac{x(\lambda-1)}{\ln \lambda} u(x),
\end{aligned}
$$

This (8) is called: the distribution of prime numbers Fundamental Theorem. density function $\mathrm{u}(\mathrm{x})$ is called.

For example: Let $\mathrm{x}=256, \mathrm{a}=16$, [a $/ 2]=8, \lambda=1.41421356$, by (8) get:

$$
\begin{aligned}
& \quad \frac{x(\lambda-1)}{\ln \lambda}=\frac{256(1.41421356-1)}{\ln (1.42421356)}=305.9629, \\
& \begin{aligned}
u(256)=\sum_{181 \leq p \leq 256} \frac{1}{\lambda p}+\sum_{128 \leq p \leq 181} \frac{1}{\lambda^{2} p}+\cdots \cdots+\sum_{16 \leq p \leq 22} \frac{1}{\lambda^{8} p} \\
\quad=0.0389781+0.0354226+0.0230385+0.0196627 \\
\quad+0.0129908+0.0093341+0.0097421+0.0069659 \\
\quad=0.1561
\end{aligned} \\
& \text { get } \quad s(256,16)+\pi(16)=305.9629 \cdot 0.1561+6=54-0.23919131 .
\end{aligned}
$$

Actual $\pi(256)=54$,

The calculations show that ignore 0.23919131, Integer 54, And the actual number is equal. $\mathrm{x}$ is the greater, the smaller the remainder. 
By (8) get :

$$
\pi(x)=s(x)-s\left(x^{1 / 2}\right)+\pi\left(x^{1 / 2}\right)
$$

By (9) Convert:

Thus:

$$
\begin{array}{r}
\mathrm{s}(\mathrm{x})<2 \pi(\mathrm{x}), \\
s\left(x^{1 / 2}\right)<2 \pi\left(x^{1 / 2}\right),
\end{array}
$$

By (9) get:

By (10), (11) get

$$
s(x)-s\left(x^{1 / 2}\right)<\pi(x)<s(x)-s\left(x^{1 / 2}\right)+2 \pi\left(x^{1 / 2}\right),
$$

$$
s(x)-2 \pi\left(x^{1 / 2}\right)<\pi(x)<s(x)+2 \pi\left(x^{1 / 2}\right),
$$

By (12) get The prime number theorem.

\section{Mertens Theorem}

In 1874 , mathematician Mertens prove[5] $]_{11}$

$$
\sum_{p \leq x} \frac{1}{p}=\ln \ln x+A_{1}+O\left(\frac{1}{\ln x}\right),
$$

This (13) is: Meitens Theorem.

Set $\ln x \rightarrow \infty$, by (13) get

$$
\sum_{p \leq x} \frac{1}{p}=\ln \ln x+A_{1},
$$

by (14) get

$$
\sum_{x i-n \leq p \leq x \lambda-n-n} \frac{1}{\lambda^{n} p}=\frac{1}{\lambda^{n}} \ln \ln \left(x \lambda^{1-n}\right)-\frac{1}{\lambda^{n}} \ln \ln \left(x \lambda^{-n}\right)=\frac{\lambda^{-n} \ln \lambda}{\ln x-n \ln \lambda},
$$

by (15) Substituting (8), get:

$$
s(x)=x(\lambda-1) \sum_{n=1}^{a} \frac{\lambda^{-n}}{\ln x-n \ln \lambda}, \quad a=\left[\frac{\ln x-1 / 2}{\ln \lambda}\right], \quad \lambda=\sqrt[x / 2]{x},
$$

this (16) and $\mathrm{Li} \mathrm{(x)}$ are 1/lnx integrator. Therefore:

$$
\operatorname{Li}(x)=s(x)
$$

For example: Let $\mathrm{x}=100000000, \lambda=1.0000003684136828, \mathrm{a}=48642829$,

By (16) get $\mathrm{s}(100000000)=5762209$,

By (2) get Li (100000000) = 5762209,

\section{Proved a prime number theorem}

By (12), (17) get:

$$
\operatorname{Li}(x)-2 \pi\left(x^{1 / 2}\right)<\pi(x)<\operatorname{Li}(x)+2 \pi\left(x^{1 / 2}\right), \quad(x \rightarrow \infty) .
$$

This (18) is a new prime number theorem. Is equivalent to the ideal formula of Riemann.

\section{To continue the discussion}

After reading this, I believe that the Fundamental Theorem already have a clear understanding of the distribution of prime numbers. Theorem density from Gauss to the regional distribution of prime numbers, Fundamental Theorem to the distribution of prime numbers. Then prove a new prime number theorem.

Get a function equal to $\pi(\mathrm{x})$ for the distribution of prime numbers, many complex issues, such as Jie Bove conjecture, Goldbach conjecture, twin primes conjecture, Riemann Guess would like to become clear through the end, easy to understand. 
Riemann formula is an approximation to $\pi(\mathrm{x})$ function, its discovery and proof is so difficult. You can imagine, it is a function of $\pi(\mathrm{x})$ is equal to what is not easy to discover and prove. Even if you want to get a $\pi(\mathrm{x})$ is equal to the conjecture is not easy.

By (8) Can also be obtained from :

$$
\pi(x)=s\left(x, x^{1 / 3}\right)+\pi\left(x^{1 / 3}\right),
$$

For sufficiently large $\mathrm{x}$, another new prime number theorem:

$$
\operatorname{Li}(x)-2 \pi\left(x^{1 / 3}\right)<\pi(x)<\operatorname{Li}(x)+2 \pi\left(x^{1 / 3}\right),
$$

This is beyond this topic, Not be discussed in detail.

Thanks: Chinese Taipei, Mr. Chen, Professor Qin Ji. Huazhong University of Science and Technology Professor Lu Yuanhong. Zhao Lu, Shandong Zaozhuang II teacher computer programming.

\section{References}

[1] Manin (Russia) and other. (2006). modern number theory guided the Science Press.

[2] Hua.(1979). number theory guide .Science Press.

[3] (Germany), Neukirch.(2007). Algebraic Number Theory. Science Press.

[4] Hua, Wang Yuan.(1963). numerical integration and its application. Science Press.

[5] Pan Cheng-dong, the Pan Chengbiao.(1988). prime number theorem, elementary proof of the .Shanghai Science and Technology Press.

[6] Lu Changhai.(2004). (USA). Riemann hypothesis.

[7] Dan Liu.(2011). Distribution of prime numbers iterated function.Canadian mathematics research.

[8] Dan Liu.(2012). Conversion of the Riemann prime number formula. Asian Journal of Mathematics (SAJM) 